\title{
La "Nueva Arquitectura" financiera y las búsquedas del Banco Mundial
}

\section{Introducción}

En este fin de siglo se ha hablado de "crisis" hasta la saciedad, pero no deja de sorprender que los abanderados del neoliberalismo mundial en su última reunión entonaran un himno en todo de "mea culpa". ¿Estamos ante una proclamación oficial de la crisis? ¿Es tiempo de anunciar el fin del neoliberalismo o la crisis de las instituciones que lo gerencian? ¿Se divisan serios propósitos de enmienda por parte de quienes reconocen los errores? Es lo que pretenden desentrañar los autores en este artículo al filo del discurso del Presidente del Banco Mundial en octubre pasado a los países miembros del mismo Banco en abril. Tomando en serio las palabras de ambas personalidades -señores Wolfensohn y Stiglitz- se trata de reflexionar desde la filosofía y la economía, simultáneamente, por dónde debería transitar realmente cualquier intento de salir de esa crisis, a fin de no comenzar el próximo siglo con el "más de lo mismo" que nos mantiene en permanente aflicción ( $y$ que ahora, parece, empieza a afligirlos también a "ellos").

\section{Desarrollo}

El último trimestre del año de 1998 nos sorprendió con el Mitch, la detención del general Pinochet, los bombardeos de Estados Unidos a Irak, y la declaración del Presidente del BM sobre la crisis económica y humana que afecta al planeta entero. Desde hace ratos nos sabemos en crisis, pero el tono de alarma del discurso del máximo responsable de tan benemérita institución levanto cierta polvareda en los círculos políticos y económicos,' por lo menos en la medida en que las palabras la pueden levantar. En el discurso del presidente del $\mathrm{BM}^{2}$ es de señalar en primer lugar el tono de preocupación personal que se refleja, que no es el de la inquietud del tecnócrata por el comportamiento de variables macroeconómicas que desde su perspectiva son controlables, sino la de "ciuda- 
dano de la tierra" o de la "aldea global" preocupado porque algo inesperado e impredecible esta sucediendo ante lo cual urge hallar soluciones inéditas. En segundo lugar hay que notar que el texto ha dado la vuelta al mundo bajo el título de "la otra crisis", pero que su contenido no aclara directamente cual "la una" (crisis) y cual "la otra", aunque es de suponer que "la" crisis primera debe ser la que ha traído de cabeza este año recién pasado al sector económico y financiero internacional: la crisis del sudeste asiático, el desmoronamiento de la economía de Rusia y la precariedad de los sistemas financieros en las llamadas economías emergentes, y que "la otra crisis" es la crisis humana que experimenta la población del planeta y la biosfera en general bajo tales efectos. Al leer a Wolfensohn da la sensación de que acaba de descubrir que las víctimas tienen nombre y rostro humano, y que se siente moralmente impelido a constituirse en una especie de divulgados de las esperanzas frustradas de quienes esperaban ver, una vez caídos los muros y devueltas las democracias a su lugar, un tercer milenio que se iniciara, si no con prosperidad, por lo menos con la sensación de estar adecuadamente en el camino de las transformaciones económicas que hicieran realidad el sueño de los economistas: el desarrollo.

Pero la realidad, la evidencia empírica, ha hecho despertar a todos de un sueño de poco más de diez años de triunfalismo, cuando las víctimas e, incluso, los que no han sentido y sienten tan de cerca los efectos devastadores de esa crisis, ya se están empezando a preguntar ante la vista de lo que acontece y con ojos atónitos, qué pasa con las promesas de la modernidad; qué de la organización y modernización de los estados nacionales; cómo es que la universalización de la medicina lo único que ha hecho posible es mitigar el dolor de millones de seres que esperan silenciosamente la muerte; en fin, en dónde está, se preguntan, la "vida mejor" tantas veces prometida. Todo esto sobre lo cual el señor Wolfensohn reflexiona en voz alta, pareciera que es nuevo, pues reconoce que las propuestas en las que tenía fe no han funcionado, y estima que la alocución del presente año (i.e. 1998) se dan en "una coyuntura mundial muy distinta a la del año pasado». ${ }^{3}$

\section{Wolfensohn ¿De la esperanza a la crisis en un año?}

¿Qué ha pasado para que el señor Wolfensohn se exprese de esta manera? ¿Pueden los países cambiar de un año a otro de una situación de bonanza, de equilibrio, a otro de incertidumbre? ¿Puede ser tal la magnitud de una crisis para modificar situaciones en tan breve tiempo? ¿Cabe argüir desconocimiento de los dinamismos de la mundialización ante la supuesta imprevisibilidad de la crisis? ¿Qué fuerza oculta del mercado es capaz de generar una crisis no pronosticable como la que el Presidente de este organismo que por más de cincuenta años -y no desde hace uno o dos- determinó y condicionó el comportamiento de la economía? Las respuestas a estas interrogantes no habría que buscarlas en los instrumentos utilizados para procurar el crecimiento de los paises, que no hacen 
sino medir el ritmo esperado de "desarrollo" o lo que es lo mismo, el comportamiento de las variables macroeconómicas partiendo de los propios supuestos del modelo. Las respuestas, más bien, habrá que buscarlas en los efectos secundarios que aquellas ocasionan en los sectores de la economía y agentes económicos que están excluidos del patrón de crecimiento. ${ }^{4}$ Lo correcto por lo que se pregunta implícitamente el señor Wolfensohn tiene que ver precisamente con lo que las más de las veces no constituyen prioridades para el Banco, ${ }^{5}$ específicamente con el entorno político y social, aspectos éstos que quedan desplazados de las decisiones de política económica, salvo cuando razones tiene que ver con la seguridad de las grandes potencias. ${ }^{6}$ No por gusto este señor reconoce que "con demasiada frecuencia nos hemos centrado excesivamente en lo económico, sin comprender bien los aspectos sociales, políticos, ambientales y culturales de la sociedad". ' Sin embargo esta aseveración, junto con el propósito de enmienda de buscar un enfoque menos restringido, tiene un resabio inevitable de "excusa de mal pagador". ¿Cómo se puede alegar desconocimiento de los vínculos entre macro y microeconomía, o incluso sobre los dinamismos de la mundialización para disculpar la falla de que las propuestas económicas no han sido todo lo "integrales" que se requiere cuando precisamente las Naciones Unidas" se han destacado por trabajar sistemáticamente con una alta compartimentación hasta el punto de que las misiones de funcionarios de "alto nivel" no se consideran como "políticas" por el mero hecho de no proceder directamente de un estado?"

Por otro lado, resulta paradójico y hasta curioso ver cómo reaccionan estos organismos internacionales y los países que se constituyen como los principales socios (accionistas "mayoritarios" del banco) para acudir en defensa de las economías que más interesan al sistema intemacional. Evidentemente, se observa un trato diferenciado en el tipo de exigencias que imponen a los diferentes países. Así, cuando se acudió al rescate de Rusia del caos económico que le sobrevino a su crisis política, lo que más importó a occidente fue exigirle medidas de privatización generalizadas que encontraban más justificación en los intereses relativos al equilibrio político-militar que en resolver los problemas económicos que la aliviarían de las presiones internas. No se buscó ahí como involucrar la economía rusa dentro de la competencia intemacional poniendo énfasis en la modernización de su capacidad de producción. El mayor interés de occidente en este caso fue buscar como neutralizar el poderío militar ruso que se concentraba en empresas bajo el control del estado, razón suficiente para condicionar toda la ayuda a las transformaciones del aparato productivo bajo la creencia de que con ello se lograban los propósitos estrategico-militares. No sólo en este contexto el caso ruso merece atención especial, sino también porque con el desmembramiento de la ex-Unión Soviética se desmoronó paralelamente el CAME (Consejo de Ayuda Mutua Económica, bloque económico cuya versión militar lo representó el pacto de Varsovia), esa especie de autarquía con la que estos estados enfrentaron el largo período de la Guerra Fría. En contraste con ello, en el caso 
de china, país con un modelo de economía centralizada análoga a la rusa y caracterizada también por su potencia de demanda, se ha insistido en la apertura a la modernización de su capacidad de producir sin que ello comportara la exigencia de políticas de privatización. Entre estos dos ejemplos tan significativos se encuentran los países en desarrollo, que no vienen sino a constituir una especie de "reservas menores" para las actividades productivas y comerciales de la economía globalizada. Cabe incluso un detalle adicional: mientras que en países como China o Rusia hay mayor interés en promover la base económica con su componente de apoyo financiero, en los países en desarrollo se prioriza el movimiento de capitales, sin prácticamente ninguna preocupación por la transformación de las bases productivas. La principal lección que proporciona la experiencia rusa está en el hecho de que no obstante el patrimonio cientificotecnológico -incluyendo en ello el poderío nuclear- existe todo un pueblo que está envuelto en el pesimismo, un pais desgastado y sin vicios de futuro. Y en esto, no se diferencia de cualquier país del Tercer Mundo atraído gradualmente por la globalización de los mercados y alimentados con la euforia de mantener equilibrios macroeconómicos, aunque los índices crecientes de desempleo los hagan aterrizar en la realidad.

Después del "período de esperanza", que es como se califica en el documento aquel lapso de tiempo en que el BM y FMI conjugaron esfuerzos para implementar a fondo las medidas de corte neoliberal, dice Wolfensohn que "llegó un año de turbulencia y confusión". Para el presidente del Banco Mundial vendría a ser un "annus horribilis", pero en sí no explica qué es lo que le ha abierto los ojos, es decir, si reacciona por simple temor a la inestabilidad originada en las principales bolsas de valores que llevó hasta el suicidio a unos cuantos corredores de bolsa japoneses (por vergüenza de su fracaso como líderes, o por el descubrimiento de su corrupción) o lo hace como reconocimiento implícito de que la aplicación y el tratamiento de las recetas monetaristas no se pueden hacer de manera uniforme en todo tiempo y lugar y que no dejan de tener unos "efectos - sociales- secundarios" imposibles de ocultar. Son seguramente estos efectos los que inducen al presidente del Banco a declarar que se está en un estado de emergencia social.

\section{Estado de emergencia social y reformismo}

Sin embargo, ya sea por levantar cargos respecto a esa responsabilidad reconocida o por aceptación de que tiene que tomarse un camino distinto hacia el desarrollo, el hecho ès que el señor Wolfensohn remarca que se está dedicando especial atención a la "adopción de medidas de corto y largo plazo para la recuperación sostenida" "'I refiriéndose con ello a las iniciativas impulsadas por la institución que preside para el fortalecimiento de los marcos legales e instituciones que sirven de soporte a la modernización de la economía. Al respecto, mientras que un conjunto de estas reformas se orientan, según el texto al campo 
normativo-financiero y judicial, buscando básicamente crear un mayor clima de confianza en el sector privado, en particular al inversor extranjero, otras constituyen reformas en el campo social, principalmente en aquellas áreas que tienen que ver con la educación y la salud, como instrumentos para crear entre las comunidades pobres mayores oportunidades de acceso al desarrollo. Pero, es necesario denunciar que estas iniciativas que en efecto han venido impulsándose desde hace años en algunos países (y también a instancias de estas instituciones, hay que reconocerlo), no han tenido mayor impacto porque están enmarcadas dentro de políticas macroeconómicas que no tienen como prioridad, por ejemplo la promoción del empleo. Así, mientras las recetas fondomonetaristas insistan en manejar políticas restrictivas de la demanda, con un control también estricto del gasto público, y pugnen por mantener ante todo niveles bajos de inflación, poco se podrá avanzar en materia de desarrollo humano; o, para usar las palabras del propio presidente del Banco, "de colocar en primer plano a las personas"."

Para el Banco Mundial el asunto de la sostenibilidad se plantea como el reto de conjugar simultáneamente tres elementos: la modernización de la economía, su crecimiento sostenido y la equidad (aunque de manera muy parcial) en la distribución del producto de dicho crecimiento. Sin embargo, mientras el Banco mantenga el divorcio entre estos tres elementos, la exclusión de amplios sectores de la sociedad será una constante; ya que el tratamiento del asunto no radica en su simple identificación como problemas, tomados aisladamente, sino en su forma de estar relacionados. Al Banco Mundial, tanto como al Fondo Monetario Internacional y otras instituciones análogas, les cuesta reconocer que su visión del desarrollo es esencialmente excluyente. En otras palabras, que el modelo sólo funciona para sectores modernos de la economía; esto es, para los sectores en los cuales el "mercado" aplica, o ha tenido que incorporarlos por necesidad. En concreto, se "moderniza" lo ya existente sólo para acondicionarlo a las necesidades del presente; creciendo sostenida y equilibradamente sólo aquella "porción" de la economía que se adecua a las reglas del mercado restringido —básicamente financiero-, pero sin permitir que se incorporen nuevos sectores $y$, lo que es más, sin una tendencia a que se integran nuevos mercados, a escala nacional. En esta "dinámica", las reformas sociales - llámense educación o salud- no constituyen sino una especie de seguro mínimo e indispensable para garantizar la reproducción ("sana" y "capacitada") de aquel ámbito restringido de mano de obra que el mercado operante demanda.

En general, las políticas que han impulsado estos organismos han estado lejos de propiciar la integración de las economías (así, en plural) al interior de los países a los que han llegado, supuestamente, como salvadoras. Y menos se puede decir, como corolario de ello, que hayan podido impactar positivamente en la mejora de los niveles de vida de las poblaciones. Deben reconocer que entre ellos mismos han sido, y aún son, incapaces de articular e integrar sus recetas como para presentarlas, por los menos, de manera coherente. Por lo 
tanto, poco podemos esperar que logren resultados favorables. El término "equilibrio" es sólo aplicable dentro de su concepción, a lo económico. El resto -esto es, lo social, lo político lo cultural- son aspectos secundarios que condicionan - "distorsionan", siguiendo su lenguaje ya clásico-, pero no determinan el comportamiento de las relaciones económicas Por eso se limitan a hablar solo de reformas. No existe para ellos nada que, estructuralmente, represente la necesidad de una transformación profunda de la sociedad, pues el sistema de propiedad, la cuestión de la redistribución internacional de las ganancias, la consideración seria del desgaste ecológico, etc. siguen, y seguirán siendo para ellos cuestiones intocables. La corrupción, el narcotráfico, la impunidad, incluso la violación a los derechos - a todos los derechos- de las personas, no representan dentro de su marco de análisis sino "distorsiones" propias de sociedades con economías retrasadas. En su credo basta el mercado para "corregir" estas distorsiones, por ello la preocupación actual del Banco por apoyar proyectos de reformas políticas se orienta básicamente a proponer marcos legales de corte netamente punitivo y "reformista", la gobernabilidad democrática deja de constituirse, al menos para nuestros países, en un modo, un estilo de vida, de convivencia social, para limitarse a un conjunto de normas que proyecten al inversor extranjero una "imagen-país" propicia -es decir, garantizable- para sus inversiones y la adecuada repatriación de los beneficios obtenidos. Se llega hasta callar ante las dictaduras, tanto abiertas como solapadas, con tal que exista un orden que dé garantía al capital extranjero. Total, las reformas, en caso de llegar a realizarse, representan puras aplicaciones de maquillaje que ni siquiera alcanzan el rango de operaciones de cirugía estética.

Sin embargo, a pesar de que los juicios anteriores provienen de la evidencia empírica del modo de actuar de los organismos financieros internacionales (IFIs) no se puede desdeñar ni tomar con ligereza cualquier intención legítima -ojalá sea así; y de serlo, habría que apoyarla - que tenga el Banco en pretender una transformación de las sociedades "subdesarrolladas". Pero para tomar esta nueva actitud como precursora de una auténtica reflexión de cambio, será necesario rescatar la tesis de la "nueva arquitectura"/2 distanciándola de aquella otra creada hace cincuenta años, que el mismo presidente del Banco reconoce que no ha logrado resolver todos nuestros problemas. En efecto, cuando el señor Wolfensohn señala en su discurso que "el desarrollo es algo más que ajuste"1.3. no hace sino poner el acento en que la nueva arquitectura que urge crear va más allá de lo financiero. Se trata, remarca, de la necesidad de un "segundo esque$m a$ ", un nuevo enfoque que incluya "la dimensión humana y social", incorporando en ello "la conservación del medio ambiente, la condición de la mujer, el desarrollo rural, las poblaciones indígenas, el progreso en materia de infraestructura". ${ }^{14}$ Es decir, una reforma que realmente lo sea, integrando todos los campos de la vida humana, proponiendo cambios estructurales y quebrando por lo menos cierto número de tabús (o simplemente poniéndolos sobre el tapete). Tam- 
bién será interesante prestar atención a ese llamado que hace para iniciar un debate en torno a lo que ha sido un tema tabú - el dogma - para los defensores del sistema: el cuestionamiento de la arquitectura financiera que ha estado vigente por casi cincuenta años. Obsérvese que por primera vez, quizás, un organismo como el Banco tiene la osadía, si no de contraponer, por lo menos de situar en un mismo plano de discusión la dinámica de la estructura financiera con lo que él llama los "fundamentos del desarrollo". 's Y es tal la relevancia que le otorga a este debate que lo lleva a identificarlo como condición para superar la "crisis silenciosa" que se proyecta en el horizonte. No se trata pues, como se resaltó anteriormente aludiendo a análisis superficiales de las crisis financieras actuales, de un asunto de "distorsiones del mercado" sino de algo más sustantivo.

Pero para ponderar la fe que podamos tener en los tales "cambios" o "reformas", tal vez valga la pena insistir en los intentos de corrección, anteriores y actuales, a los que se somete el sistema para enfrentar las distorsiones que afectan el modelo. En un momento lo fue el llamado "consenso de Washington" "l" tendiente a corregir - en los ochenta - los efectos de los excesos de endeudamiento en los que cayeron los países subdesarrollados durante la época de los "estatismos" (década de los sesenta). Y hace dos años, lo ha sido el "consenso de Santiago" (abril de 1998), en el que a nivel de la Cumbre de las Américas ha surgido la inquietud — todavía "inqúietud"- respecto a cómo enfrentar los efectos de las recetas correctivas de la crisis de la deuda. Curiosamente, los resultados derivados de las políticas de ajuste que han dado pie a las preocupaciones del "consenso de Santiago", es decir, la urgencia de asumir las responsabilidades sobre los asuntos del crecimiento con equidad, el narcotráfico, la corrupción, el medio ambiente, etc., han sido calificados con un eufemismo casi vergonzoso: "dureza del ajuste". No se precisa que los resultados obtenidos por la "dureza del ajuste" son, ni más ni menos, los efectos lógicamente esperados de las políticas de ajuste estructural, propias de un modelo cuya dinámica, principalmente en relación a los países subdesarrollados, se sostiene en tres ejes: un economicismo excluyente de posibilidades y oportunidades para amplios sectores de la población; formas de gobernabilidad adecuadas puramente a los intereses del momento; y, políticas irracionales en el uso y preservación de los recursos naturales y ambientales. Es importante caer en la cuenta que el "consenso de Santiago" es tomado más como un llamado para buscar salidas aisladas a los temas de actual preocupación - nuevamente aquí el eufemismo de las "distorsiones"-, que la urgencia por corregir los efectos directos del "consenso de Washington". Y como si fuera poco, en esta actitud frente a una crisis real del modelo de desarrollo vigente $^{17}$, que ya evidencia impactos perversos en las economías pobres, no se duda en hacer convivir - llamémoslo así- un consenso con el otro. Un ejemplo de esto es el Brasil de Cardoso, heredero e impulsor a su vez, él mismo, de la extrema pobreza que convive con la corrupción, la violencia (que llega hasta el 
asesinato de niños de la calle), crisis de instituciones democráticas, etc. ¿Cuál ha sido la respuesta a su actual situación de crisis financiera? Sencillamente, acudir a salvar el modelo mediante las recetas propias del "consenso de Washington", dejando de lado las "inquietudes" del "consenso de Santiago". ${ }^{\prime 8}$ Y esto, a pesar de que un conspicuo representante del Banco Mundial, como lo es el señor Stiglitz - nada menos que su Vicepresidente y Economista en Jefe- enfatiza que la "continua adherencia ciega al consenso de Washington limita nuestro potencial para el desarrollo". 14

Definitivamente, es obvia la ruptura - $i$ divorcio? - que, por lo menos en el discurso, existe entre el Banco Mundial ${ }^{211}$ y el Fondo Monetario Internacional. ${ }^{21}$ Habría que darle al primero el beneficio de la duda, en el sentido que habiendo participado seguramente en la construcción del consenso de Washington, está tomando distancia de él, y acercándose a las preocupaciones (es mejor llamarlas asî) del consenso de Santiago. Más bien de lo que no cabe duda es que, habiendo sido el FMI el artífice del primer "consenso" no estuvo de acuerdo - aunque posiblemente estuvo presente- con el segundo. Esto es más obvio, cuando el Sr. Stiglitz comete la osadía —así tomarán sus apreciaciones los del FMI- de cuestionar, tomando como referencia la evidencia empírica, algunos de los dogmas de los recetarios del Fondo, como: los "costos" de la inflación, cuando esta se sitúa por debajo de determinados niveles; determinación de niveles "óptimos" de déficits fiscales y cuenta corriente; los costos de la estabilización macroeconómica, frente al crecimiento a largo plazo ${ }^{22}$; los límites de la competencia y el libre comercio ${ }^{23}$; las privatizaciones apresuradas sin creación de estructuras institucionales adecuadas (mercados competitivos y regulaciones); involucramiento del Estado; etc. ${ }^{24}$

\section{El mea culpa de Stiglitz: ¿enfoque crítico o pura evasión de responsabili- dad?}

Entre los varios puntos que señala Stiglitz como errores del enfoque destaca en primer lugar que el control de la inflación no es lo más importante para que la economía tenga buena salud. "Es posible que ni siquiera sea un componente esencial", dice. ${ }^{25}$ Por otra parte sostiene que "el enfoque de la liberalización de mercados puede haber tenido un efecto perverso de contribuir a la inestabilidad económica al debilitar el sector financiero". La tercera afirmación es que el consenso de Washington no dio la importancia que merece a la producción y al desempleo en vistas a la promoción de crecimiento a largo plazo, especialmente en los países de desarrollo, al tiempo que denuncia que en el consenso de Washington la liberalización comercial y economía en general y el control de la inflación y de los déficits fiscales son fines en sí mismos. Pero se cuida muy bien de no apuntar específicamente los efectos "inequitativos" que ello ha producido. Asimismo asevera que la "competencia es imperfecta, especialmente en los países en desarrollo". ${ }^{2 h}$ Es su modo de expresar la realidad: así como llaman 
"distorsiones del mercado" a las medidas gubernamentales de los países para proteger mínimamente a las economías locales, llaman "imperfección de la competencia" a las redes de acaparamiento en el tercer mundo y a los nuevos desafíos anticompetitivos que plantea por ejemplo Bill Gates en el primer mundo. Por otra parte también se posiciona contra el neoliberalismo al señalar que "el estado tiene un importante papel que jugar en la regulación apropiada, la protección social y la seguridad social", ante lo cual nos surge la duda de sí verdaderamente fueron ellos o no (evidentemente no solos, sino con "los grandes", léase el G-7) los creadores o implementadores de la teoría y la práctica del desmantelamiento del estado. ${ }^{27}$

El nuevo enfoque que el Banco Mundial intenta dar a la "ayuda al desarrollo" para ser realmente novedoso tendría que enfrentarse con las terribles disparidades sociales inducidas por las llamadas "políticas de estabilización", porque si bien no puede francamente responsabilizarse a éstas por su creación ${ }^{28}$, si hay que mantener enfáticamente que las han agudizado. Para comprobar esta afirmación puede tomarse como referencia la variable "consumo", como un indicador que mide el impacto de los beneficios inducidos por el libre comercio de mercancías y de capitales a escala mundial. La realidad contrasta fuertemente con los postulados teóricos de quienes defienden y negocian estas posiciones cuando se observa el abismo existente entre el grado de desarrollo de los países dueños de capitales y la tecnología (incluyendo los mecanismos del comercio) y los países proveedores de recursos primarios y de mano de obra barata. ${ }^{29}$ Estas serias contradicciones de la globalización reducida a vínculos de comercio y de libertad plena de movimientos de capitales, se destacan en el último Informe sobre Desarrollo Humano ${ }^{30}$ publicado por el Programa de las Naciones Unidas para el Desarrollo (PNUD). En efecto, el Informe señala que la quinta parte de la población más rica consume el $45 \%$ de la carne y el pescado extraído, mientras que la más pobre, sólo el $5 \%$. Esa misma población más rica consume el $58 \%$ de la energía producida, mientras que la más pobre consume menos del $4 \%$; la más rica consume el $84 \%$ del papel producido, y la más pobre el $1.1 \% .^{31}$ Asimismo, se señala que el $20 \%$ más pobre de la población mundial "ha quedado excluido de la explosión del consumo". Y que decir que la calidad de vida si más de 2,600 millones de personas de los países en desarrollo carecen de saneamiento básico. Esta mundialización de la pobreza se refleja, además, en la existencia de 2000 millones de personas en estado anémico. Y junto a estos abismos en el consumo, el Informe señala que el deterioro ambiental y el agotamiento de los recursos renovables han ingresado a una fase peligrosa, y toda la responsabilidad de esta situación recae inequívocamente en los países altamente industrializados. Así, ilustrando lo anterior, "un niño nacido en el mundo industrializado agrega más al consumo y la contaminación a lo largo de su vida que entre 30 y 50 niños nacidos en países de desarrollo." 32 
Si los defensores radicales del libre mercado calificaron a los 80 como la "década perdida" debido al estancamiento de las economías subdesarrolladas como consecuencia de las drásticas medidas que tuvieron que aplicarse para "corregir" los errores del "estatismo" de los 70, ¿cómo llamarían - si es que se atreven- a la década de los 90 en las que muchos países empiezan a ver que sus esfuerzos y sacrificios por un desarrollo armónico y sostenido se han reducido a un sueño; y otros tantos, que ya ven agotadas sus posibilidades de sobrevivir ante la crisis? ¿Es esta la situación sustentada en la experiencia concreta que obliga al señor Wolfensohn a referirse al hecho de que "la aldea está en llamas"; que lo lleva al punto de declarar la urgencia de buscar respuestas inmediatas, ${ }^{3 .}$ porque si no se logra "una mayor equidad y justicia social, no habrá estabilidad política"? ?4

El reto que impone la mundialización como una realidad que nos supera no está ni en la concurrencia irreflexiva e inercial de quienes la reducen a prácticas y mecanismos de mercado ni en las actitudes absurdas de aquellos que pretenden evadirlas volviéndose hacia el interior de las fronteras nacionales. Si ya no es posible negar que los mercados están cada vez más vinculados e integrados y que por eso mismo, las crisis financieras tienden a trasladarse; menos podrán negarse los efectos de exclusión que la dinámica concentradora del capitalismo neoliberal provoca. Así como se mundializan los mercados, haciéndose uno; de la misma manera, la pobreza va adoptando un mismo rostro a escala planetaria. Precisamente, porque el Banco Mundial, en la persona del señor Wolfensohn, reconoce que al armar y sostener la arquitectura financiera no se penso en los elementos vulnerables de la economía, en su sostenibilidad y en una transformación socio-económica duradera, la pobreza ha conducido a una verdadera crisis humana. Mientras los capitales y las mercancías se desplazaban entre los países -estratégicamente situados- ${ }^{35}$ en procura de optimizar las tasas de ganancia, la pobreza iba en aumento en razón de la carencia de oportunidades de acceso a una actividad que provea de ingresos mínimos, traduciéndose en desplazamientos masivos de personas desde los países pobres hacia los desarrollados, ${ }^{36}$ en conflictos territoriales con origen en la necesidad de sobrevivencia y en violencia y desbordes sociales crecientes.

Frente a la situación de crisis ${ }^{37}$ "de la población mundial", la tesis de la nueva arquitectura que Stiglitz y Wolfensohn levantan más bien desde una perspectiva de desarrollo humano adquiere mayor relevancia cuando, equivocadamente -o tercamente, en sus posiciones- los defensores del modelo de la "vieja" arquitectura financiera, intentan situar la crisis asiática o la rusa -y en general, cualquier otra que podría surgir en otro momento ${ }^{38}$ como un asunto de "distorsiones del mercado". Incluso, los críticos que se sitúan en el lado opuesto -incluidos los izquierdistas "pragmáticos" de ahora- caen en este juego, cuando analizan los fenómenos actuales buscando razones o explicaciones en los efectos de dichas crisis y no en sus causas. No se trata, hay que decir, de 
analizar si los capitalistas se mueven o no libremente, sino dentro de qué ámbito y bajo qué reglas de juego lo hacen, en escala mundial. El mercado, como pretenden los teóricos del liberalismo, no es equivalente al concepto geométrico de locus, ni lo puede explicar todo como el éter a los antiguos astrónomos. El mercado es un ámbito concreto de las relaciones humanas en donde los recursos naturales, humanos, financieros, tecnológicos se asignan también con criterios políticos, sociales, culturales, ambientales. Es un ámbito en el que se mueven intereses de todo tipo.

En este sentido Stiglitz va algo más allá que Wolfensohn al señalar puntos bastante más concretos de lo que él estima son "fallos no previstos" en las recetas neoliberales, llegando a admitir incluso que el éxito de crecimiento entre 1978 y 1995 corresponde a un país socialista: China. Parece pues que el BM está realizando una cierta autocrítica respecto a sus propias políticas; sin embargo, habría que preguntarse qué potencial crítico tiene todo ello. No cabe esperar grandes cambios de actitud de quienes habitualmente se han caracterizado por decir las verdades a medias y cuando la revelación de estas verdades resulta del todo inocua por no ser susceptible de provocar ningún cambio en la realidad. Stiglitz destaca, como decíamos, el fracaso del consenso de Washington; pero el solo hecho de presentamos como un gran cuestionamiento novedoso (como quien descubre el agua tibia) que el "desarrollo" debe ser algo más que puro mercado dejado a su inercia con la mínima regulación estatal, nos hace caer en la sospecha de que pocos cambios buscan. La bibliografía sobre el desarrollo es extensa y variada y no sólo en América Latina donde se puede hablar de una cierta tradición, ${ }^{34}$ que no data solamente de años recientes. La propia ONU en sus agencias y programas utiliza esquemas de desarrollo de "avanzada" que, de alguna manera, recogen actualmente dicha tradición. ${ }^{411}$ Entonces, ¿cómo es posible que diga que los temas de "equidad, medio ambiente, los efectos distorsionantes del crimen y narcotráfico constituyen temas relativamente nuevos para economistas tradicionales?"4l Es difícil ver ahí algo distinto de una flamante evasión de responsabilidad.

\section{6. ¿Buscando el control del descontrol?}

No deja de ser llamativo que uno de los puntos en torno al cual se hace más acalorada la discusión sea el tema del control del sistema financiero, pues se estima que "sin el marco legal apropiado, los mercados de valores simplemente no cumplen sus funciones vitales en detrimento del crecimiento del país a largo plazo". También es de notar que Stiglitz viene a decir que "el fin (la competencia) no justifica los medios (la privatización a ultranza) porque al fin y al cabo -y sobre todo en el tercer mundo- no hay una sólida institucionalidad y no se trata de una competencia entre iguales. De ahí que la solución que propugnan estribará en la implementación de mayores reglas y controles; algo así como un intento de reconducir el juego para restablecer el "fair play" sin cuestionarse 
para nada sobre qué tipo de juego es éste que cada vez descarta a más personas. ¿Puede alear el sistema "inocencia" ante un tipo de descontrol que es el puro efecto de su dinámica? En esta línea pueden ser muy verdaderas sus preocupaciones por buscar el control del sistema financiero que dentro de la dinámica de la mundialización escapa cada vez más a normas de cualquier tipo, pero ¿no se está de algún modo proponiendo controlar lo incontrolable? Además se habla de control del sector financiero sin abordar el problema de la vinculación con la economía real. Sin enfocar directamente estos problemas mal podremos hablar de desarrollo.

Por otro lado resulta que la competitividad sigue siendo el objetivo principal para alcanzar una economía "moderna", pero la privatización no es el único medio para llegar a ésta. Parece ser que esta novedad se desprende de las experiencias de Rusia y China, pues dice al respecto: "las experiencias de Rusia y China presentan dilemas para las teorías económicas tradicionales", pero de ahí no desprende Stiglitz que tal vez valga la pena cuestionarse la privatización ni que quizás capitalismo y desarrollo no van necesariamente de la mano, pues esto sería hacer una aseveración política y desbordaría los límites de un trabajo que pretende ser técnico. Seguramente también sería extralimitarse decir que ambas experiencias nos hacen también reflexionar sobre el tema de la equidad. Frente a este modo de tratar los problemas hay que señalar que la "nueva arquitectura", si pretendiera ser nueva, más global y verdaderamente humana no podrá plantearse en términos puramente teocráticos, sino que tendrá que considerar en primer plano los planteamientos sociales y políticos de la población del planeta. Será imposible hablar de equidad si conservamos las mismas estructuras políticas internacionales en las que los que tienen más mandan más y aún reciben más. El propio Stiglitz alude indirectamente a esto cuando dice que el consenso de Washington quiso proporcionar una fórmula para fortalecer el sector privado y estimular el crecimiento que esta fórmula no resultó, y que por ello hay que crear un nuevo consenso que no puede estar basado en el de Washington. Uno de los principales motivos que aduce es que "los países en desarrollo no pueden reclamar estas políticas como suyas", pero no dice claramente porqué: porque hunde cada vez más a las mayorías, porque estos marcos económicos obligan a vender al país, a hipotecarlo, cosa que no es algo muy honesto hacer. Se trata, en definitiva, aunque no lo reconozca, del problema "Norte-Sur", de la división entre ricos y pobres y de un sistema que tiende a aumentar las diferencias económicas.

La "novedad" de estos planteamientos está en que el discurso "oficial" ha pasado de la línea de un "capitalismo mesiánico" de finales de los 80 a otro de corte más moderado, pero no cabe albergar grandes esperanzas de cambio en la política de quienes pontifican sobre cómo debería construirse una economía que funcione sobre la base en la que ha venido funcionando. Admiten que hay descontrol, pero pretenden que la lógica del sistema lo pueda corregir, sin apre- 
ciar que las crisis pertenecen a su ausencia. Hasta ahora desde los púlpitos de la economía moderna se consideraba que los males "colaterales" que se observaban eran males necesarios para llegar a una mayor bonanza económica. Parece que ahora el aumento a todas luces de la concentración de bienes, del desempleo, que queda consagrado como un mal inevitable estructuralmente, y la multiplicación de la crisis, ponen en cuestión que todo vaya para bien. Pero se sigue confiando en que estas tendencias se pueden revertir sin quebrar el sistema. Por todo ello no cabe sino mantener ante las políticas económicas para frenar el descontrol de una economía global así entendida bien vale un voto de esperanza -aunque no de confianza- venga de donde venga. Así como el caso Pinochet ha abierto un debate y estudio de las instituciones jurídicas internacionales, así también cabe la posibilidad de que en una de estas coyunturas inesperadas surjan propuestas inéditas. Todo depende de la fuerza que logren levantar los sectores interesados en los cambios y del consenso que logren articular en torno a las alternativas. Aquí hay una doble lucha: la del diálogo, la de las ideas, la de la profundización y cuando esta no vale de la fuerza. No la fuerza militar, por supuesto, sino la que surge del decir: señores hasta ahí llegamos. ¿Qué ha pasado con la Cumbre de Río?, ¿con el feminismo? Vale argumentar; pero donde los argumentos no hacen mella, lo efectivo y necesario es obligar a la negociación, a "firmar acuerdos". Tampoco los sindicatos en la Europa del bienestar se formaron porque los empresarios se empaparon de las ideas ilustradas, sino por la conciencia o el activismo, como se le quiera llamar, de la clase obrera. Porque ellos y ellas dijeron: hasta ahí llegamos. Por esto es necesario como tercer mundo fraguar alternativas en todos los frentes o dimensiones de la realidad. Afilar los conceptos, las ideas, pero también recrear una internacional de la "buena voluntad", hacer un ecumenismo del respeto humano que se enfrente a todo tipo de fundamentalismo bloqueador o continuista.

Es duro decirlo pero la actitud que asumimos frente a esta nueva postura que deja traslucir el Banco Mundial, pareciera mostrar que se trata de que quienes están sentados en la misma mesa son el escéptico y al cínico, de la filosofía. Es decir, de un lado, quienes buscan promover una mundialización de dimensión realmente humana, fundamentada en principios de equidad y solidaridad en el uso de los recursos terrestres y del conocimiento; y, de otro, quienes reiteradamente - históricamente- no hacen sino reproducir aquellas relaciones de desigualdad entre los pueblos a escala planetaria. Viene a ser la confrontación entre el que duda —el escéptico- pero que no por ello elude el diálogo, con el que lo niega todo -el cínico - protegido cómodamente en sus dogmas. Si por lo menos, haciéndose consecuente con aquel eslogan que destaca en su documento sobre un mundo libre de pobreza, el Banco Mundial realmente pasara de una actitud cínica a otra escéptica, creemos que mucho habremos avanzado y, sobre todo, poder acoger con esperanza y disponibilidad los discursos de sus representantes visibles. 
La evidencia empírica nos pone ante la realidad de una globalización que se va tejiendo paradójicamente, bajo los parámetros actuales, de manera excluyente, reproduciéndose en dicho proceso la vieja pero no por ello agotada o superada - muy a nuestro pesar- dicotomía dominador-dominado. Comparativamente y guardando las distancias históricas, las relaciones que se tejen actualmente a nivel mundial bajos los mecanismos de mercado, concebidos y aplicados de acuerdo a ámbitos y reglas específicas que no son impuestas desde los organismos supuestamente creados para la promoción humana, hacen caer en la cuenta que se trata de viejas ataduras con nuevos nudos.

Sobre estas bases tenemos que planteamos si la economía del tercer milenio tiene que estar fundamentada en los mismos conceptos o es hora de revisarlos para poder plantear una nueva economía política. Si lo que interesa son puras reformas ya podemos ir haciéndonos la idea de que nos va a tocar "más de lo mismo". Desde esta perspectiva el problema no estaría tanto en la "falta de autoridad" 42 o de "solvencia moral de los que mandan", ni siquiera en la creación de instituciones que se hagan cargo de la economía global, sino en ponernos de acuerdo sobre lo que ha de ser económica y políticamente - por lo tanto respecto al plano humano- la eficacia económica y hacia que clase de sistema debería de evolucionar el capitalismo para ser mas equitativo. Esto tiene que ser ampliamente sujeto a debate. Se trata ni más ni menos de abordar el sempiterno problema de la justicia. A estas alturas no basta adoptar un tono de lamento moralista como el que exhala el discurso del presidente del BM que nos lleva a pensar que no busca ningún compromiso o alternativa concreta ni mucho menos un cambio. $Y$ es que redistribuir a estas alturas de apertura del tercer milenio no va a significar solamente - que ya seria mucho- compartir los recursos económicos, sino desconcentrar el poder político, tecnológico, científico, cultural de occidente por una razón de seguridad. Así como el nacimiento de las democracias nacionales está ligado a la división de poderes, así un concierto mundial tendrá que estar necesariamente comprometido con la desconcentración del poder político. Si no se da esto corremos el peligro de ir a parar a un totalitarismo global. Esta crítica, este debate es algo que no es susceptible de hacerse sin levantar algunas ampollas. En otras palabras, supone estudiar y debatir a fondo los problemas de la producción-productivismo, de la ciencia-cientismo, del empleo y el ocio, de la educación y del analfabetismo, del medio ambiente; y ello repitámoslo una vez más- buscando los modos de revertir políticamente la tendencia centrífuga del sistema a la concentración de poder, admitiendo que seguramente nadie tiene "la" razón, pero sí un cúmulo largo de experiencias que prudentemente analizadas algo pudieran aportar si hubiera la suficiente "buena voluntad". Sin este debate y la confianza en que algo puede cambiar la acción humana, tendremos unos efectos inciertos de un capitalismo imprevisible que no por ser el viejo capitalismo de siempre deja de tener aspectos totalmente inéditos que desconciertan hasta a sus más fervientes defensores; pues a medida que 
crece la vinculación sistemática entre la sociedad planetaria, crece también la velocidad e imprevisibilidad de los cambios.

Ojalá esta "crisis silenciosa" o más bien apabullante desde los marginados, la de los huracanados de siempre... ${ }^{43}$ sirva para entablar una cruzada de diálogo, de buena voluntad. Ojalá sea una crisis humana que revierta en una nueva esperanza. Y esto no se consigue sólo haciéndonos propósitos de ser todos muy buenos, sino haciendo algunas reformas estructurales efectivas para que podamos ser mejores. De no ser así la riqueza y la pobreza continuarán siendo cada vez más las principales señas de identidad de los sobrevivientes del genocidio (desaparición física sistemática y selectiva) de los pueblos del planeta.

\section{Notas}

1. ECA se hizo eco de la temática de análisis dedicándole un amplio editorial, así como dos artículos, que sin ceñirse ni con mucho a este documento abordan el tema del neoliberalismo, la globalización y la necesidad de buenas dosis de creatividad para enfrentar los retos que plantea la coyuntura de cara al tercer milenio. Las referencias al texto del discurso están tomadas de la versión publicada en ECA № 600 Año LIII, UCA San Salvador, octubre 1998 págs. 1003-1009

2. El texto lleva el título sugerente de "La otra Crisis" y estuvo dirigido por el Sr. James D. Wolfensohn, Presidente del Grupo del Banco Mundial a la Junta de Gobernadores que es la máxima instancia del Banco —equivalente a lo que en una empresa sería el Directorio- y compuesto por los representantes (denominados "gobernadores") de los 178 países miembros. Cuando en su alocución el señor Wolfensohn utiliza la expresión "Señor Presidente" lo hace para referirse específicamente al máximo representante de esta Junta, nombrado para presidir la reunión. Cabe señalar también que cuando se alude al "Grupo" del Banco Mundial la referencia está dada para el conjunto de las cuatro instituciones que lo conforman: el Banco Internacional de Reconstrucción y Fomento (BIRF), al que le corresponde contribuir a la reconstrucción y el desarrollo de los países miembros; la Asociación Internacional de Fomento (AIF), cuya tarea es la concesión de prestamos a los países más pobres; la Corporación Financiera Internacional (CIF), cuya finalidad es la brindar asistencia técnica y financiera al sector privado con la condición de que contribuya al desarrollo del país; y el Organismo Multilateral de Garantía de Inversiones (OMGI), al que corresponde facilitar (garantizar) la corriente de inversiones privadas con fines productivos hacia los países miembros.

3. Dice textualmente: "Hace un año la producción mundial crecía a un ritmo del 5.6 por ciento —el más alto de los últimos 20 años. Hace un año, Asia oriental experimentaba dificultades, pero nadie preveía la gravedad de la caída. Hace un año Asia meridional, donde vive el $35 \%$ de los pobres de todo el mundo, no conocía todavía las pruebas nucleares, y parecía que podría disfrutar de un crecimiento del 6 0por ciento en los años futuros. Quizás más. Hace un año, los países en desarrollo habían emprendido un camino que prometía un crecimiento sostenido durante el próximo decenio. Hace un año había optimismo sobre Rusia y su fuerte equipo reformista" (ECA \#600 p. 1003). 
4. Es lo que Manfred Max.Necf llama "los scctores invisibles" Cfr. Economia descalza. Señales desde el Mundo Invisible Cepaur-Nordan, Bogotá, 1984 p.41.

5. A pesar de que dicho organismo se creó hace más de cincuenta años con el objetivo de reducir la pobreza y mejorar los niveles de vida mediante la promoción de crecimiento y el desarrollo económicos sostenibles.

6. Por ejemplo, el caso de Rusia.

7. Eca NN $^{2} 600$ octubre p. 1006

8. A todo esto vale hacer recordar que si bien el BM, creado hace 50 años, no cs propiamente un organismo integrante del Sistema de las Naciones Unidas, cstá adscrito a él, y tiene como objetivo reducir la pobreza y mcjorar los niveles de vida mediante la promoción del crecimicnto y el desarrollo económico sostenibles.

9. Al rehusar el calificativo de políticas (tanto las "misiones" como los funcionarios) hace que se separen con mayor facilidad temas que van inevitablemente de la mano según la cconomía política, para presentar como puramente técnicas un buen númcro de acciones que en realidad no solo son porque no cmanan de los cstados.

10. Eca № 600 pág. 1007

11. Eca № 600 pág. 1004

12. Eca № 600 pág. 1009

13. Eca № 600 pág. 1006

14. Eca No 600 pág. 1007 ss.

15. Eca $\mathrm{N}^{\circ} 600$ pág. 1008

16. Véase Más Instrumentos y Metas más amplias: desde Washington hasta Santiago. Stiglitz, Joscph. Traducción autorizada para el Proyecto Estado de la Nación, del trabajo presentado en el seminario "Estabilidad y desarrollo cconómico en Costa Rica: las reformas pendientes”; San José, Costa Rica, abril de 1998. El señor Stiglitz es Vicepresidente y Economista en Jefe del Banco Mundial, y también autor, junto con David Sappinton, del «Teorema fundamental de privatización" (1987).

17. En su versión de crisis financiera internacional, "rusa" y "asiática".

18. Es la expresión acuñada por el Sr. Wolfensohn en su discurso ante la Segunda Cumbre de las Américas (Santiago, 18-19 de abril de 1998), en razón de las preocupaciones sobre ciertos asuntos que afectan al mundo y en particular el continente (crecimiento sin equidad, deterioro ambiental, violencia, corrupción, narcotráfico, etc.); y, como consecuencia de ello, de los compromisos que asumieron los paises del continente americano respecto a darle al desarrollo un carácter que vaya más allá de lo estrictamente económico y financiero. Precisamente, el título de la conferencia del Presidente del BM fue "The Santiago Consensus - From Vision to Reality" (Santiago, april, 1998. Tomado de página web del BM).

19. Véase Más Instrumentos y Metas más amplias: desde Washington hasta Santiago. Stiglitz, Joseph Opc. Cit. P 4

20. Cabe aquí hacerse la pregunta si cuanto funcionario de alto, medio y bajo nivel -y principalmente aquéllos que viajan de misión a los países- estarán concientizados con esta "nueva" visión del BM, que coloca a este organismo, en las personas de los señores Wolfensohn y Stiglitz, en una posición de vanguardia.

21. A pesar de que también tiene sus ramalazos críticos, como cuando su director gencral, Sr. Camdesus afirmó que "hemos pasado de un fundamentalismo de Estado a un fundamentalismo de Mercado". 
22. Al respecto, Stiglitz destaca: "Irónicamente, la estabilización macroeconómica, según la conceptualización del consenso de Washington, típicamente le resta importancia a la producción y al desempleo". En: Más Instrumentos y Metas más amplias: desde Washington hasta Santiago. Stiglitz, Joseph. Op. cit. P.9

23. "La liberación comercial, sin embargo, no es ni necesaria ni suficiente para establecer una economía competitiva e innovadora", dice. Ibid. P.15

24. Cf. Más Instrumentos y Metas más amplias: desde Washington hasta Santiago. Stiglitz, Joseph. Op. cit.

25. Stiglitz p.6

26. Stiglitz p.20

27. Hace un par de meses, sin ir más lejos -y después del paso del Mitch- han felicitado al presidente de Nicaragua, Dr. Arnoldo Alemán por ser buen alumno en esta asignatura, y como premio se le condona el $80 \%$ de la deuda.

28. Nos referimos en este aspecto a las diferencias producidas como resultado de estas políticas; lo que no significa que las disparidades no tengan su origen en la lógica capitalista.

29. De acuerdo al esquema neoclasico, es de suponer que todos los países que están integrados a los flujos "globalizados" de comercio de bienes/servicios y de movimientos de capitales globalizados tienen a beneficiarse mediante el incremento en sus niveles de consumo e inversión; lo que cquivaldría a una mejora significativa en la calidad de vida, vía mayor empleo e ingresos.

30. Informe sobre Desarrollo Humano 1998. Programa de las Naciones Unidas para el Desarrollo (PNUD). Ediciones Mundi-Prensa; Madrid, 1998.

31. Informe sobre Desarrollo Humano 1998. Op. cit., pp 2 y 3

32. Ibid. P.4

33. "Ser audaces, pero también realistas", acota (p.4).

34. Eca No 600 p. 1009

35. Por ejemplo, para el capital norteamericano, desde el punto de vista financiero, no cs lo mismo China Popular que Filipinas; o México con respecto a Centroamérica.

36. Incluso se presenta el mismo fenómeno entre países más pobres hacia aquéllos de desarrollo relativo superior, o internamente, desde zonas rurales a urbanas.

37. "Es mucho lo que está en juego, demasiadas vidas humanas", indica. Y como respondiendo a una realidad distinta a aquella que envuclve a csta forma exclusiva y excluyente de concebir la globalización (cn lo cconómico-financiero), el Presidentc del Banco Mundial califica a la actual crisis como una "crisis humana" (Eca \# 600 p. 1009) que afecta a todos. $Y$ va a remarcar esto enfatizando repetidamentc que "mientras los mercados se desploma y las cifras de la pobreza se disparan", no se podría estar debatiendo sobre una nueva arquitectura (financiera), desatendiendo, de paso, las urgentes demandas de los paises en desarrolio en un mundo cada vez más interdependiente.

38. En su oportunidad, vale recordar, lo fueron la crisis polaca y la mexicana; y como ahora, en razón de que por su posición estratégica dentro del sistema internacional pusieron en peligro la estabilidad financicra, Estados Unidos y otros países del Grupo de los Siete (G-7) no dudaron en acudir prestos a inyectar en sus cconomías ingentes recursos.

39. Sólo para señalar aquéllos que prescntan carácter integral en su concepción del desarrrollo, se pueden citar los trabajos de L.J. Lebret, Manifiesto por una civiliza- 
ción solidaria; Ed Universo, Lima 1960 y Dinámica concreta del desarrollo; Herder, Barcelona 2da. Ed. 1969; y el de Denis Goulet Etica del desarrollo, Estela-Cepal Barcelona 1965.

40. Destacan en esto los Informes sobre el Desarrollo Humano que el Programa de las Naciones Unidas para el Desarrollo (PNUD) vienen publicando anualmente, desde 1990. Recuérdese además que tanto el BM (o BIRF Banco Internacional de Reconstrucción y Fomento) como el FMI (Fondo Monetario Internacional) son organismos adscritos al Sistema de las Naciones Unidas y, por tanto, no le deben ser ajenos los diferentes trabajos que se han producido.

41. Stiglitz p. 3. El llamado problema Norte-Sur se viene abordando desde hace 20 años, y el "nuevo orden económico internacional" que acabó poco más o menos que en papel mojado, surge con una declaración de la ONU de 1994.

42. Eca $\mathrm{N}^{\mathrm{Q}} 600$ octubre 1998, p. 880

43. No es hiperbólico hablar de la "injusticia del cambio climático", pues los efectos sobre el clima también dividen a la humanidad entre el $80 \%$ que emite el $20 \%$ de los gases invernadero y el otro $20 \%$ que es responsable de la emisión del $80 \%$ de los gases. No sólo se reparte injustamente la responsabilidad de originar los cambios de clima sino el sufrimiento de sus efectos. Los impactos no son del mismo calibre en los países ricos que en los pobres. 